\title{
A spectroscopic study of the Themis family
}

\author{
M. Florczak ${ }^{1,2}$, D. Lazzaro ${ }^{1}$, T. Mothé-Diniz ${ }^{1}$, C.A. Angeli ${ }^{1}$, and A.S. Betzler ${ }^{1}$ \\ 1 CNPq/Observatório Nacional Depto. Astrofísica, 20921-400 Rio de Janeiro, Brazil \\ e-mail: florczak@on.br, lazzaro@on.br \\ 2 CEFET, Depto. Física, 80000 Curitiba, Brazil
}

Received July 3; accepted September 4, 1998

\begin{abstract}
We present spectroscopic observations of 36 asteroids, members of the Themis family. These observations were carried on at the European Southern Observatory in the wavelength range $4900-9200 \AA$. Most of the objects present a spectra similar to C-type asteroids and some of them present indicative of aqueous alteration. We discuss the implications of these results on the formation and evolution of this family.
\end{abstract}

Key words: minor planets, asteroids

\section{Introduction}

Themis is one of the most statistically reliable family in the asteroid belt. First discovered by Hirayama (1918), along with Eos and Koronis, it has been identified as a family in all subsequent works (see Zappalà et al. 1995, and references therein). According to a modern view (Farinella et al. 1992) the term "family" should be attributed only to clusters of asteroids whose physical properties support a common origin. Thus, the study of surface composition of Themis members is fundamental to define or, at least, constrain its collisional origin. Furthermore, if there is no doubt, from a statistical and physical point of view, that these are indeed fragments resulting from the breakup of a parent-body, thus important constraints on the latter can be obtained. The information about whether the parent-body was differentiated or primitive will lead, consequently, to constraints on the primordial temperature of the solar nebula which are fundamental for a correct modelling of the Solar System formation.

\footnotetext{
* Based on observations made with the $1.52 \mathrm{~m}$ telescope at the European Southern Observatory (La Silla, Chile) under the agreement with the $\mathrm{CNPq} /$ Observatório Nacional.

$\star \star$ Table A1 is available at the CDS via anonymous ftp 130.79.128.5 or http://cdsweb.u-strasbg.fr/Abstract.html

$\star \star \star$ Appendix A is only available at the journal,

http://www.edpsciences.com
}

A very striking feature of Themis family is its closeness to the border of the 2/1 mean motion resonance, as shown by Morbidelli et al. (1995). This resonance corresponds to the Hecuba gap, the widest of all the gaps in the asteroid belt, on which much work has been done in the last years trying to understand its depletion mechanism (see Henrard et al. 1995; Morbidelli 1996; Moons 1997; Ferraz-Mello et al. 1998). According to Morbidelli et al. (1995), when the Themis family is plotted in an appropriate resonant phase space, its rightmost limit is coincident with the border of the $2 / 1$ resonance. As a consequence they suggest that the catastrophic event which gave rise to Themis family might once have populated the resonance zone. Due to some depletion mechanism all the objects were then swept off this region, creating the gap. If this assumption is true, then it implies that fragments, similar to those present today in the Themis family, might have been "transported" to other regions of the asteroid belt. It has also been suggested (Dermott et al. 1992, and references therein) that collisions in the Themis family could contribute to the formation of the Zodiacal Cloud. A precise surface characterization of asteroids members of Themis family can, therefore, help to investigate the fate of fragments injected in the Hecuba gap.

In the present paper we report spectroscopic observations of 36 asteroids, members of the Themis family, and discuss the implications of these results on the formation and evolution of this family. In the next section we present a short characterization of the Themis family and discuss some hypothesis on the fragmentation which gave origin to it. The observations and data reduction are presented in Sect. 3 along with a discussion of their implications on the origin and evolution of the family. A summary of the main results of the present work is given in the last section. 

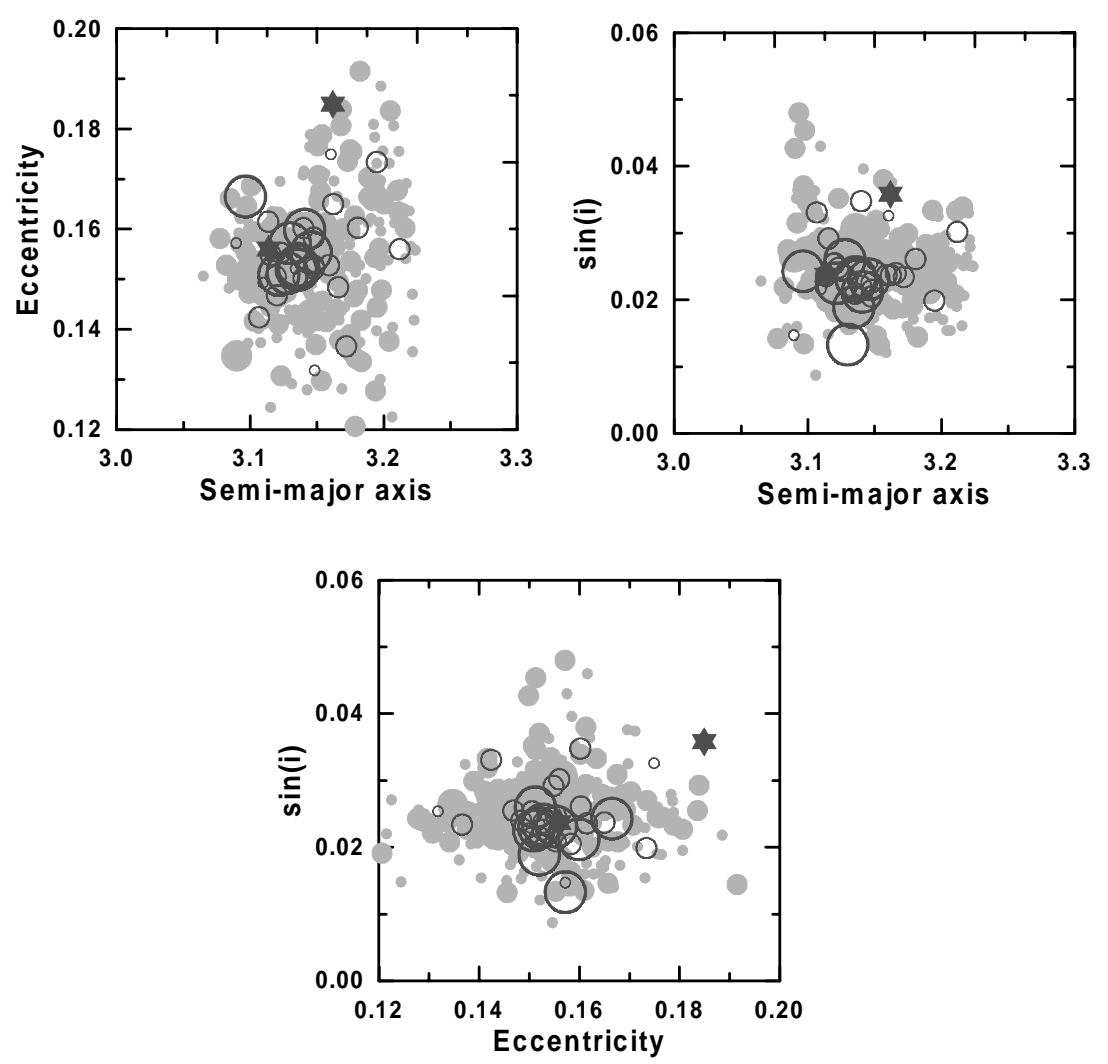

Fig. 1. Distribution of the Themis family in proper elements phase space: a) semi-major axis $\times$ eccentricity, b) semi-major axis $\times$ sine of inclination, and c) eccentricity $\times$ sine of inclination. The size of the circles is proportional to the diameter of the objects and the open circles indicate those observed in the present work (available on-line)

\section{The Themis family}

Themis family is located in the outer part of the main belt with proper semi-major axis in the region from 3.06 to $3.23 \mathrm{AU}$. The orbits have relatively high eccentricities and small inclinations. The Themis family, as determined by Zappalà et al. (1995) has 550 members: four - 24 Themis, 90 Antiope, 171 Ophelia, and 268 Adorea - with a diameter greater than $100 \mathrm{~km}$, ten with diameter in the range $50-100 \mathrm{~km}$ while the remaining are smaller than $50 \mathrm{~km}$. The expected size limit of completeness for this family corresponds to $20 \mathrm{~km}$ (Zappalà \& Cellino 1995). According to Migliorini et al. (1995), the expected number of interlopers is relatively low, even if one or two can be expected at sizes as large as $40 \mathrm{~km}$.

The distributions of the proper elements in the $a-e$, $a-\sin (i)$ and $e-\sin (i)$ planes are shown in Fig. 1, using Milani \& Knezevic (1994) proper elements. In this figure the sizes of the circles are proportional to the diameter of the objects and the open circles indicate objects observed in the present work. The two stars indicate objects which might be interlopers as will be discussed in the next section. A core-halo structure, in which a dense "core" is surrounded by a "halo" of decreasing density, was initially described by Williams $(1979,1992)$. However, more recent studies do not present such a distribution but mostly a ver- tical dispersion at the border coincident with the $2 / 1$ resonance. Zappalà et al. (1995) even introduce the denomination of "tribe" for this family due to its particular space distribution.

Since the pioneer work of Hirayama (1918) it has been assumed that families of asteroids are the outcome of one, or more, large impact of a body with a smaller "projectile" leading to the complete fragmentation or to the erosion of the parent body. From a statistical point of view this hypothesis is confirmed by the clustering observed when the planetary perturbations are removed, i.e., in the proper elements phase space. In this sense Themis family is undoubtedly one of the most reliable. However, the breakup mechanism which originated any family, as well as the subsequent evolution of its fragments, still rely on a wide set of working hypotheses and initial parameters which may not be the real ones (see, for example, Farinella et al. 1982; Fujiwara 1982; Zappalà et al. 1984; Davis et al. 1985; Farinella \& Davis 1992; Bottke et al. 1994; Marzari et al. 1995). Notwithstanding the limitation of these models, some hypotheses on the Themis family formation are mostly accepted: i) The parent body was completely disrupted; ii) The family is the outcome of a very energetic collision, probably unique in all the asteroid belt, with original parent body and projectile of $380 \mathrm{~km}$ and $190 \mathrm{~km}$, respectively (Marzari et al. 1995); iii) Such a 
collision has a very low probability to occur over the age of the Solar System; iv) The large remnants are probably "rubble-piles" shaped by their self-gravitation (Fujiwara 1982; Farinella et al. 1982). The mineralogical characterization of Themis family members' surfaces can, therefore, help to constrain the collisional model of its formation.

\section{Observations and data reduction}

The observations were carried on at the European Southern Observatory at La Silla (Chile) using a 1.5-m telescope equipped with a Boller and Chivens spectrograph and a CCD $2048 \times 2048$ pixels with a readout noise of \pm 7 electrons. A grating of $225 \mathrm{gr} / \mathrm{mm}$ with a dispersion of $330 \AA / \mathrm{mm}$ in the first order was used. The CCD has a square $15 \mu \mathrm{m}$ pixel, giving a dispersion of about $5 \AA /$ pixel in the wavelength direction. The useful spectral range is about $4900<\lambda<9200 \AA$ with a FWHM of $10 \AA$. The spectra were taken through a 5 arcsec slit oriented in the East-West direction. The slit width has been chosen in order to minimize the consequences of atmospheric differential refraction and reduce the loss of light at both ends of the spectrum. This is important since a substantial loss of light may lead to an erroneous spectral characterization of the object. Care was taken also in guarantee that the observations were made as near as possible to the meridian of the asteroid.

The spectra of Themis family asteroids were obtained in five observing runs in January/97, March/97, July/97, December/97, January/98 and March/98. The atmospheric conditions were good to excellent during all the observations. The observational circumstances, from EPHEM program (Tholen 1997), are listed in Tables 1, which shows the distance from the Sun, from the Earth, the solar phase angle, the estimated visual magnitude and the diameter. The given diameters are from IRAS (Tedesco 1997) whenever available, otherwise are estimated through the absolute magnitude and a visual albedo of 0.081 which is the mean albedo of Themis' family objects.

The spectral data reduction was performed using the Image Reduction and Analysis Facility (IRAF) package and taking much care to ensure a proper calibration of the spectra. The bias level of each night was determined through an average of the many bias images taken in the night. This "averaged bias" was then subtracted from each frame and pixel-to-pixel variations were removed dividing the resulting image by a normalized medium "flat field". The IRAF apsum package was used to sum the pixel values within a specified aperture and to subtract the background level. Wavelength calibration was performed using a He-Ar lamp, obtained several times during each night, and spectra were corrected from airmass by using the mean extinction curve of La Silla (Tüg 1977). Different solar analogs (Hardorp 1978) were observed in each observational run in order to compute reflectivities. It must be pointed out that in each night of observation were observed at least two solar analogs in order to estimate the quality of the night. The ratios between the spectra of the solar analogs for each night show no substantial variation. The influence of different solar analogs on the resulting spectra has also been checked showing differences less than $1 \% / 10^{3} \AA$. In Table 1 are given only the solar analogs used to obtain the spectra presented. All asteroid spectra are normalized around $5500 \AA$ by convention, unless otherwise specified.

\section{Spectroscopic results and discussion}

The obtained spectra, normalized at $5500 \AA$, are given in the Appendix. In these figures only one spectrum per object is given and the crosses indicate observations from the ECAS survey (Zellner et al. 1985). Most of the asteroids show similar featureless spectra, characteristic of C-, B-, F- and G-type asteroids. Two of them, 461 Saskia and 1171 Rusthawelia, display P-type spectra. In effect, Tholen (1989) also classifies the latter as P-type.

In order to compare the overall characteristic of the Themis family, a polynomial has been fitted to each spectrum and the result is presented in Fig. 2a along with ECAS spectra represented by dash lines. In this figure, spectra of a same asteroid obtained in different nights are also individually included. The figure shows a limited spread of the superficial composition among the members of the Themis family. The compositional distribution is completely contained in the distribution of the C-type class and its sub-classes B, F and $\mathrm{G}$ as can be seen in Figs. $2 \mathrm{~b}$ to $2 \mathrm{e}$. In these figures, the distribution of broadband spectra obtained from ECAS's survey for each of the above classes is plotted. This result, therefore, is consistent with a probable common origin of the observed asteroids except 461 Saskia and 1171 Rusthawelia which might be background objects. It must be recalled that in the region around 3.2 AU the $\mathrm{P}$ class objects should be abundant (Bell et al. 1989). The compositional analysis of a significative sample of Themis members, therefore, supports the dynamical/statistical indication of a common origin of the family, probably resulting from the breakup of a C-type parent body. In this scenario, the asteroids 461 and 1171 would be background objects. It is noteworthy that only 1171 Rusthawelia lies at the border of the family while 461 Saskia is contained well inside the nuclear region.

Considering that a collisional origin of the Themis family can be inferred, a question remains on whether the original parent body, or its fragments, experienced some degree of aqueous alteration. This information can set some constraints on the primordial nebula and the temperature gradient, or events, during the first stages of the Solar System formation. We recall that aqueous alteration is the low temperature $(<320 \mathrm{~K})$ chemical alteration of materials by liquid water (Vilas \& Sykes 1996, and references 

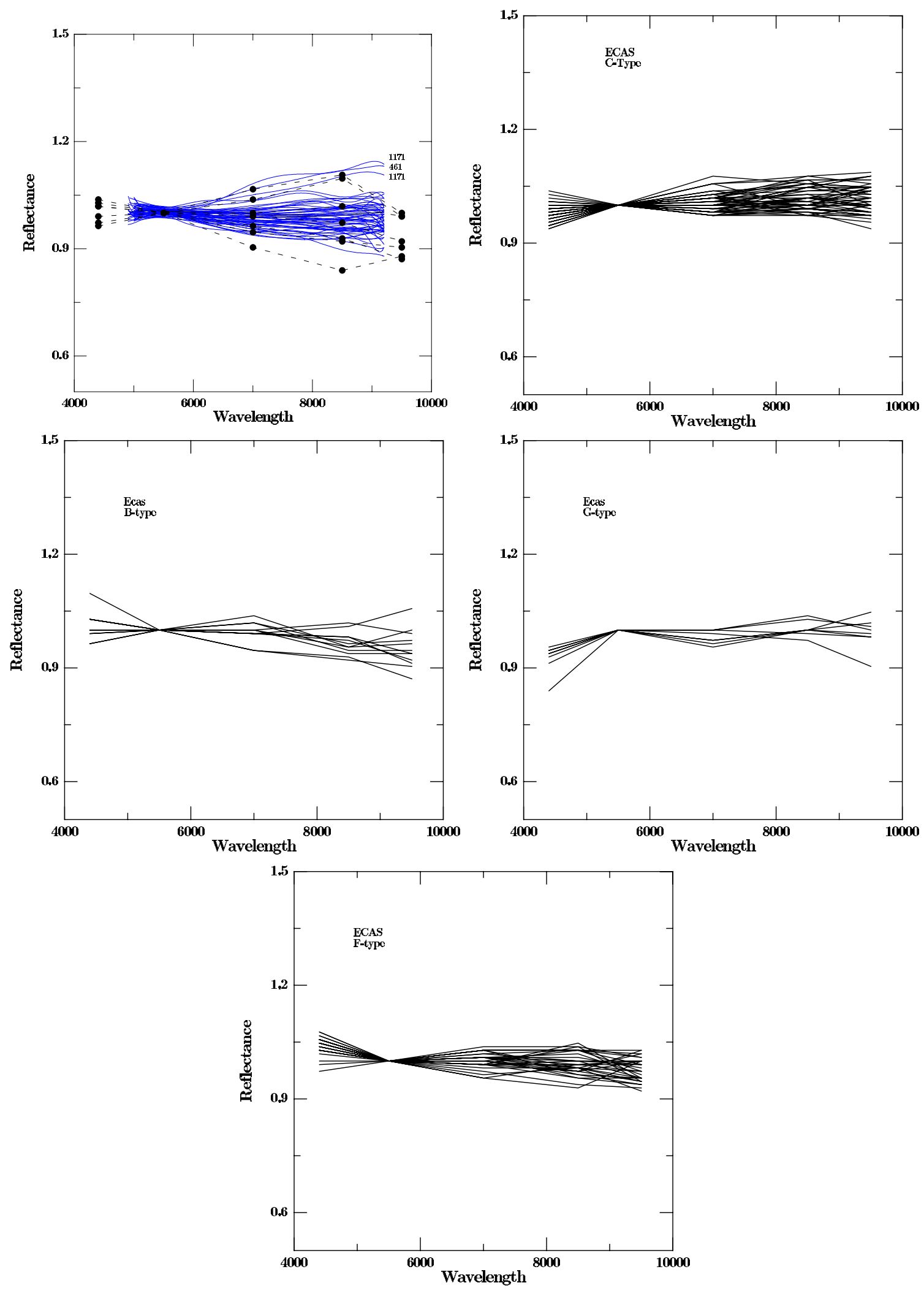

Fig. 2. a) Spectra of the Themis family presented in this work normalized around $5500 \AA$ and fitted by a polynomial for clarity along with ECAS data (Zellner et al. 1985) represented by the dashed lines. b) Distribution of broadband spectra of C-type objects obtained from ECAS's survey. c) Same as b) for B-type objects. d) Same as b) for G-type objects. e) Same as b) for F-type objects 

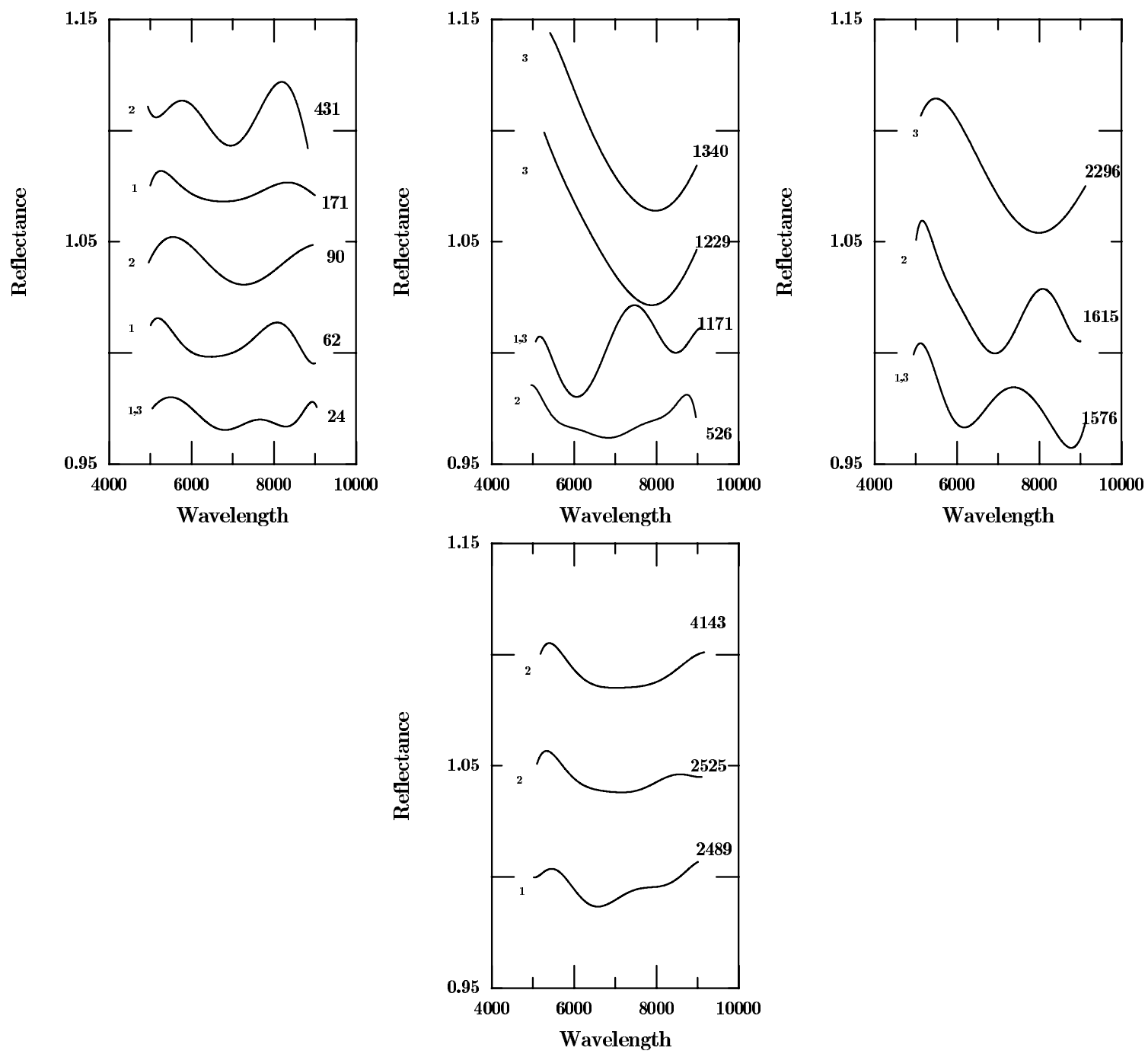

Fig. 3. Asteroids presenting absorption bands with minimum at $0.6-0.65$ (1), 0.7 (2), and $0.80-0.90 \mu \mathrm{m}$ (3), indicative of aqueous alteration in their surfaces. Each spectrum is fitted by a polynomial to increase the identification of the bands. For each spectrum is given the identified band (1,2 or 3$)$ at its left and the asteroid number at its right

therein). In order to investigate this aspect of the Themis family, aqueous alteration was searched in the spectra following the procedure described by Vilas et al. (1993, 1994). Indication of aqueous alteration, identifiable by the presence of absorption bands with minimum at $0.60-0.65$, 0.7 or $0.8-09 \mu \mathrm{m}$, is clearly present in the spectra of 15 asteroids ${ }^{1}$ among the observed ones. Since these bands are sometimes very swallow, their identification is highly dependent on the noise of the spectra so that we adopted the criterion defined by Vilas et al. (1993) in which a band is identified if its depth is greater than the peak-to-peak scatter. If a more relaxed criterion is adopted, 11 more objects could also present aqueous alteration in their surface. As

\footnotetext{
1 Asteroids 1229, 1340 and 2296 present a strong unusual absorption band between 0.8 and $0.9 \mu \mathrm{m}$, confirmed in spectra obtained in different nights, with the exception of 1229 observed once.
}

already pointed out by Vilas et al. (1993), in some cases the 0.65 and $0.90 \mu \mathrm{m}$ absorption bands seem to overlap the $0.70 \mu \mathrm{m}$ band making their identification difficult. In Figs. 3a to $3 \mathrm{~d}$ we show the polynomial fit to the spectra of asteroids that show clear indication of aqueous alteration through the $0.7,0.65$ or $0.90 \mu \mathrm{m}$ absorption band. The asteroids 316, 637, 846, 848, 954, 1487, 1539, 1691, 2519,2524 , and 3128 may also present indication of aqueous alteration but with a lesser degree of confidence and, therefore, are not included in these plots.

In Figs. $4 \mathrm{a}$ and $4 \mathrm{~b}$ we present the distributions of the observed asteroids as a function of the presence or not of aqueous alteration. In these figures the triangles and squares represent objects with and without indication of aqueous alteration, respectively. The stars represent objects that may present aqueous alteration and the size of all the symbols is proportional to the diameter of the asteroids. Considering the taxonomy of the observed objects, 

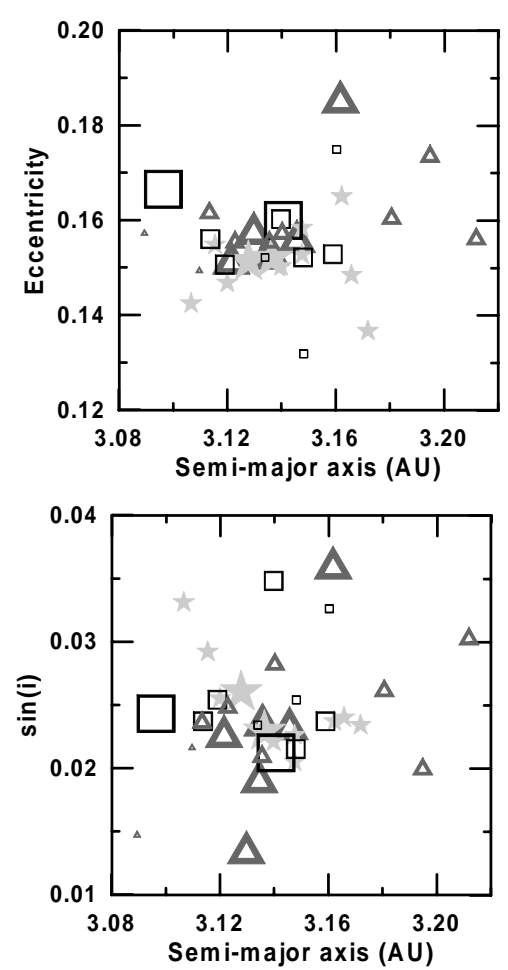

Fig. 4. Distribution, in the proper elements space, of the observed asteroids as a function of the presence (triangles) or not (squares) of aqueous alteration. The stars represent objects which may present aqueous alteration but with a lesser degree of confidence. The size of all the symbols is proportional to the diameter of the asteroids

all those classified by Tholen (1989) as F do not present indication of thermal metamorphism. This is clear in three of them: 268 Adorea, 468 Lina, and 621 Werdandi, classified as FC, CPF and FCX, respectively. Another asteroid classified as FCX, $954 \mathrm{Li}$, might or not present aqueous alteration by our analysis. These results confirm earlier suggestions (Sawyer 1991; Vilas et al. 1993; Vilas et al. 1994) that F-class asteroids may be unaltered material or that they are less or differently altered. In a recent paper, Hiroi et al. (1996) plot the UV absorption strength (the most prominent absorption band indicative of aqueous alteration) vs. IRAS diameter for the $\mathrm{C}, \mathrm{G}, \mathrm{B}$ and $\mathrm{F}$ asteroids. In their plot the F-type asteroids all present the smallest absorption band or the total absence of it. This fact is interesting since it can indicate a different evolution of this type of objects. In particular for the Themis family, it would imply a different degree of thermal alteration in different parts of the original parent body or a distinct origin of these objects. This last assumption seems not to be supported by the limited spread out in composition shown by our spectra.

Analyzing the percentage of asteroids presenting indication of aqueous alteration as a function of the diameter, a trend is apparent: it decreases with the diameter of the objects. The percentage changes from nearly $60 \%$

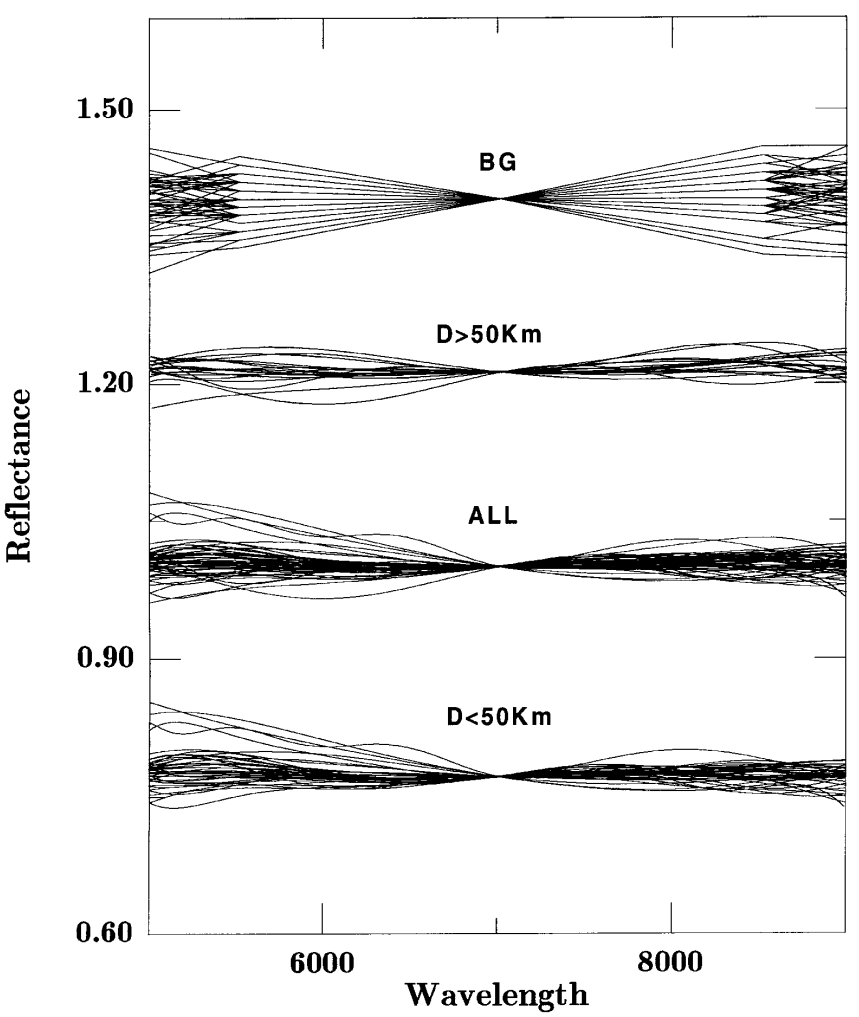

Fig. 5. Spectra normalized at $0.7 \mu \mathrm{m}$ of bakground objects from ECAS (Zellner et al. 1985), of all the asteroids observed in the present work and of the observed asteroids with diameter greater and smaller than $50 \mathrm{~km}$

to $35 \%$ for asteroids with diameter greater and smaller than $50 \mathrm{~km}$, respectively. This trend is expected by Vilas \& Sykes (1996) if we suppose the break-up of a thermally altered parent body. In their model, the bigger fragments should retain more information on the interior of the parent body where the heating event should have been more effective. If in the 11 objects which may have aqueous alteration this fact is confirmed, then the above percentages would increase to nearly $80 \%$ and $70 \%$, respectively, and the trend is less evident.

The above results tend to indicate that the parent body of the Themis family was thermally altered and the distribution of compositions can be attributed to fragments coming from different parts of the original body. Following Vilas \& Sykes (1996), if the original parent body was altered due to a thermal event in the later phases of the Solar System formation, then all the resulting fragments from a breakup should also present signs of aqueous alteration, in particular the greatest ones. This is true for the Themis family and the percentage of asteroids presenting absorption bands due to aqueous alteration decreases with the object size, as expected by Vilas \& Sykes (1996).

The dependence of the distribution of surface compositions with the diameter was further investigated. In Fig. 5 it can be seen the spectra, normalized at $0.7 \mu \mathrm{m}$, of the observed asteroids of the Themis family along with 
background objects from ECAS (Zellner et al. 1985). The figure also presents our spectra divided in asteroids with diameter greater and lesser than $50 \mathrm{~km}$, respectively. As can be seen, the distribution is slightly larger for the small asteroids. It is noteworthy to point out that this result is also expected by Vilas \& Sykes (1996). In their model the small asteroids would be fragments resulting from the breakup of a thermally altered large asteroid. Since these fragments would come from diverse compositional units of the original body, they will present a great degree of differences between them. On the other hand, the larger asteroids should be reaccreted bodies representing the original core displaying, therefore, a smaller degree of diversity.

In Fig. 5 we notice that the distribution of spectra of the Themis family is similar or slightly smaller than that of background objects. It must be pointed out, however, that a direct comparison is quite imprecise since the two types of spectra, ECAS and ours, were obtained with different techniques. A better comparison would be obtained with a background defined from SMASS (Xu et al. 1995) or from an analogous survey. Since there are too few spectra of this region in the SMASS, the real representation of the background is not guaranteed.

We also analyzed the distribution of IRAS albedo in the Themis family, where a slight correlation with the diameter seems apparent. In Fig. 6 it can be seen a larger dispersion of albedos as the objects' sizes decrease. In particular, higher albedos are displayed by smaller asteroids. However, it must be pointed out that the error bars associated to the individual IRAS albedos are greater as the diameter decreases. Taking into account this fact the trend is less apparent even if it still remains an indication, which is compatible with our spectra (Fig. 5). If this specific albedo distribution among family members is real it can imply in, at least, three distinct scenarios:

- A space weathering process acting distinctly depending on the size of the objects.

- The original parent body presented a slightly differentiated crust. In this case most of the smaller asteroids would be part of the crust presenting an albedo distinct from the bigger ones, which would be mostly reaccreted bodies from the nucleus. If this is the case, the nucleus would have an albedo around 0.02 to 0.07 , and crust values greater than 0.08. This hypothesis would imply that lighter materials present in the crust would be more reflective than the denser materials of the nucleus.

- The collision that gave rise to the Themis family occurred between a parent-body and a projectile of slightly different composition. We recall that Marzari et al. (1995) in their model of Themis' family collisional evolution, obtained the best result with a parentbody and projectile sizes of $380 \mathrm{~km}$ and $190 \mathrm{~km}$, respectively. Since the two bodies probably were not formed in the same region (otherwise the collision velocity would not lead to the catastrophic break-

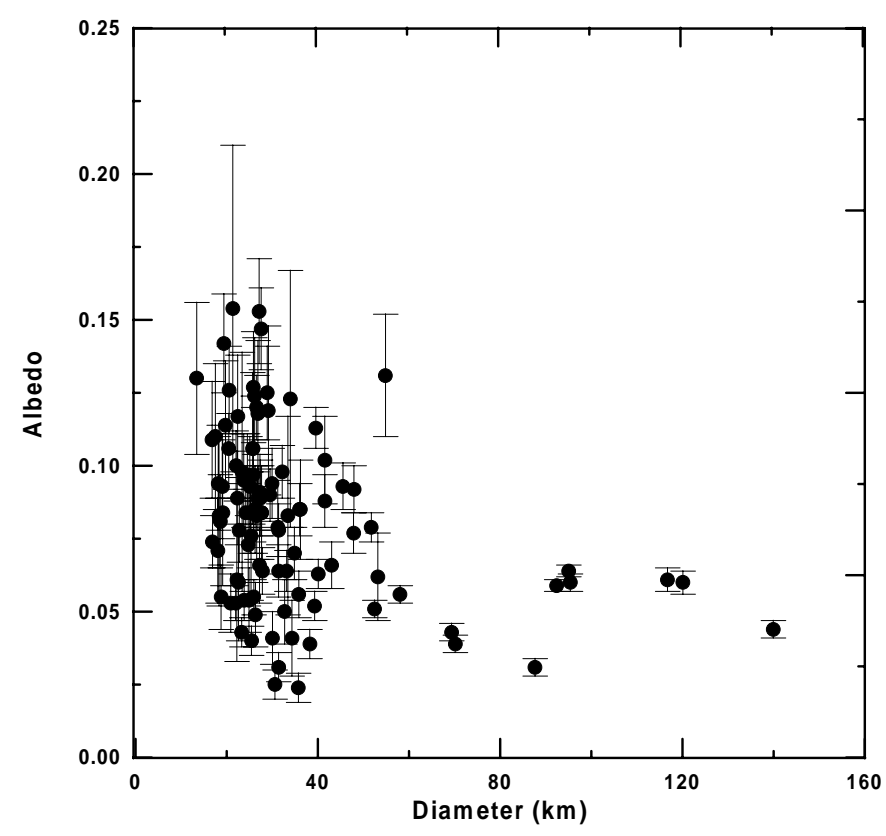

Fig. 6. Distribution of the albedos of Themis family members as a function of their diameters. A larger dispersion is apparent as the object size decreases

up of a so big object) different compositions can be expected. Moreover, since the projectile body was relatively large, its fragments would be a considerable part of the remnants. This hypothesis is, obviously, the most difficult to be accepted in view of the quite homogeneous results from the spectroscopic observations.

Among the observed objects with a known albedo, the presence of aqueous alteration was found in both low and (relatively) high albedo objects of the Themis family. Unfortunately the number of objects with available albedos and spectra is not statistically significative to permit us to find a relation between aqueous alteration and the above scenarios.

Finally the results for the Themis family were also compared to those obtained by Di Martino et al. (1997) for the Veritas family. It must be recalled that these two families occupy nearly the same heliocentric region in the outer belt. From a dynamical point of view, the greatest difference between these families is that while Themis family presents relatively high eccentricities (from .12 to .19 , proper elements) and small inclinations, Veritas family members have small eccentricities (around 0.06) but relatively high inclinations (around 10 degrees). Since the two families lie at the same heliocentric distance, it seems obvious to expect that a temperature gradient, or event, should act similarly on the two original bodies. However, a remarkable greater inhomogeneity is apparent in the Veritas family, as can be seen comparing our Fig. 5 with Fig. 3 of Di Martino et al. (1997) paper (note that the scales in the two plots are identical) Moreover, only three 
asteroids of this family seem to present indications of aqueous alteration. These results set the basis for several scenarios for the origin of the two families:

1) The heating event that thermally altered Themis original body occurred after the breakup of the Veritas family. This assumption, however, is not supported by the dynamical analysis of this family. In effect, Milani \& Farinella (1994) proved that Veritas should be one of the youngest families in the asteroid belt due to the chaoticity of 490 Veritas, as well as other members;

2) Since the original parent body of the Themis family was larger than that of Veritas (around $100 \mathrm{~km}$ ) the thermal event was more efficient in the former. This result would favor the hypothesis that the thermal event was due to the ${ }^{26} \mathrm{Al}$ decay (Grimm \& McSween 1993), since larger bodies would retain more radionuclides which would generate a greater and throughout heating of the asteroid;

3) The Veritas family was not originated from the breakup of a large body, but it would just represent a clustering of background objects. Again this assumption is not supported by the statistical clustering analysis of this family, which confirms a highly reliable Veritas family (Zappalà et al. 1995).

\section{Summary}

In this section we summarize the important points presented in this paper:

- Most of the observed 36 members of Themis family show featureless spectra similar to C-, B-, G- and Ftype. The distribution of these spectra is completely contained in the range of the above classes;

- A limited spread of the distribution of superficial composition is apparent, confirming the dynamical indication of a probable common origin from the fragmentation of a parent-body;

- Two objects, 461 Saskia and 1171 Rusthawelia, display a spectra similar to P-type suggesting that they might not be members of the Themis family but just background objects;

- Indication of aqueous alteration is clear in 15 objects and is possibly present in 11 more. This result tends to indicate that the parent-body of Themis family was probably thermally altered. This hypothesis is also supported by the larger percentage of objects presenting aqueous alteration among the big asteroids than among the small ones;

- A slight correlation between albedos and diameters is found: as the asteroid diameter decreases a larger dispersion of albedos is present and higher albedos are displayed by smaller asteroids. The same slight correlation is apparent in our spectra as discussed above.

- Comparison between Themis and Veritas families, which lie nearly at the same heliocentric distance, shows a greater inhomogeneity among the latter one. On the other hand, the presence of aqueous alteration is more prominent in the Themis family. One of the possible explanations is that since the original parent body of Themis was larger than that of Veritas a thermal event leading to metamorphism (probably due to the ${ }^{26} \mathrm{Al}$ decay) was more efficient in the former.

From the analysis of 36 members of Themis family we can conclude that a compositional homogeneity is present as would be expected from the collisional breakup of an original parent body. On the other hand, a slight heterogeneity is also apparent from the presence or not of aqueous alteration and from slightly distinct albedo populations among the family.

Acknowledgements. We acknowledge the technical staff of the ESO for their prompt help whenever needed. We are also grateful to V. Zappalà for valuable comments and remarks which much improved this paper. M.F. was supported by CAPES while D.L., T.M-D., C.A.A., and A.S.B. by CNPq, through diverse fellowships and grants. Support by FAPERJ is also acknowledged.

\section{References}

Bell J.F., Davis D.R., Hartmann W.K., Gaffey M.J., 1989, Asteroids: The big picture, in: Asteroids II, Binzel R., Gehrels T., Matthews M.S. (eds.). Univ. of Arizona Press, Tucson, p. 921

Bottke W.F., Nolan M.C., Greenberg R., Kolvoord R.A., 1994, Icarus 107,255

Davis D.R., Chapman C.R., Weidenschilling S.T., Greenberg R., 1985, Icarus 62,30

Dermott S.F., Gomes R.S., Durda D.D., Gustafson B.A.S., Jayaraman S., Xu Y.L., Nicholson P.D., 1992, Dynamics of the Zodiacal Cloud. In Chaos, Resonance and Collective Dynamical Phenomena in the Solar System, Ferraz-Mello S. (ed.), IAU Symposium 152. Dordrecht, p. 333-347

Di Martino M., Migliorini F., Zappalà V., Manara A., Barbieri C., 1997, Icarus 127, 112

Farinella P., Davis D.R., 1992, Icarus 97, 111

Farinella P., Paolicchi P., Zappalà V., 1982, Icarus 52, 409

Farinella P., Davis D.R., Cellino A., Zappalà V., 1992, A proposal for a new nomenclature, in: Asteroids Comets Meteors 1991, Harris A.W., Bowell E. (eds.). Lunar Planet Inst. Huston, p. 165

Ferraz-Mello S., Nesvorný D., Michtchenko T.A., 1998, Chaos, diffusion, escape and permanence of resonant asteroids in gaps and groups. In Solar System Formation and Evolution, Lazzaro D., Vieira Martins R., Ferraz-Mello S., Fernandez J., Beugé C. (eds.), ASP Conf. Ser. (in press)

Fujiwara A., 1982, Icarus 52, 433

Grimm R.E., McSween H.Y., 1993, Sci 259, 653

Hardorp J., 1978, A\&A 63, 383

Henrard J., Watanabe N., Moons M., 1995, Icarus 115, 336

Hirayama K., 1918, AJ 31, 185

Hiroi T., Zolensky M.E., Pieters C.M., Lipschutz M.E., 1996, Meteor. Planet. Sci 31, 321 
Marzari F., Davis D., Vanzani V., 1995, Icarus 113, 168

Migliorini F., Zappalà V., Vio R., Cellino A., 1995, Icarus 118, 271

Milani A., Farinella P., 1994, Nat 370, 40

Milani A., Knezevic Z., 1994, Icarus 107, 219

Moons M., 1997, Cel. Mech. Dyn. Astr. 65, 175

Morbidelli A., 1996, AJ 111, 2453

Morbidelli A., Zappalà V., Moons M., Cellino A., Gonczi R., 1995, Icarus 118, 132

Sawyer S.R., 1991, A High Resolution CCD Spectroscopic Survey of Low Albedo Main Belt Asteroids, Ph.D. dissertation, University of Texas

Tedesco E.F., 1997, "IMPS diameters and albedos V1.0" Planetary Data System - Small Bodies Node (PDSSBN); A'Hearn M., University of Maryland, College Park, Maryland (available http://pdssbn.astro.umd.edu)

Tholen D., 1989, Asteroid taxonomic classification, in: Asteroids II, Binzel R., Gehrels T., Matthews M.S. (eds.). Univ. of Arizona Press, Tucson, p. 1139

Tholen D., 1997, Ephemeris program EPHEM, Version 1.1, Celestech, Honolulu, Hawaii
Tüg H., 1977, Messenger 11, 7

Vilas F., Sykes M.V., 1996, Icarus 124, 483

Vilas F., Larson S.M., Hatch E.C., Jarvis K.S., 1993, Icarus 105, 67

Vilas F., Jarvis K.S., Gaffey M.J., 1994, Icarus 109, 274

Williams J.G., 1979, Proper elements, families and belt boundaries, in: Physical Studies of Minor Planets, Gehrels T. (ed.), NASA SP-267, U.S. Government Printing Office. Washington, p. 177

Williams J.G., 1992, Icarus 96, 251

Xu S., Binzel R.P., Burbine T.H., Bus S.J., 1995, Icarus 115, 1

Zappalà V., Cellino A., 1995, Main belt asteroids, In Present and future inventory, ASP Conf. Ser.

Zappalà V., Farinella P., Knezevic Z., Paolicchi P., 1984, Icarus 59,261

Zappalà V., Bendjoya P., Cellino A., Farinella P., Froeschlé Cl., 1995, Icarus 116, 291

Zellner B., Tholen D.J., Tedesco E.F., 1985, Icarus 61, 355 